\title{
Performance of transect and point count underwater visual census methods
}

\author{
R.A. Watson ${ }^{\mathrm{a}, *}$, T.J. Quinn II ${ }^{\mathrm{b}}$

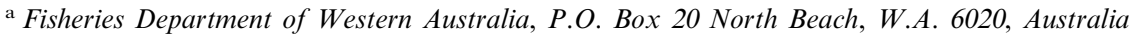 \\ b Juneau Center, School of Fisheries and Ocean Sciences, University of Alaska Fairbanks, 11120 Glacier Hwy., Juneau, \\ AK 99801-8677, USA
}

Received 18 March 1997; accepted 28 May 1997

\begin{abstract}
A simulation approach was used to study bias and variability of density estimates of fish using the transect and point count underwater visual census methods. Three experiments were conducted to examine the effects of fish density, sampling effort, and the speed of fish in relation to the observer. Fish density and sampling effort did not significantly bias estimates of fish density using either census method, and variation was a function of the area sampled with both methods. The speed at which fish approached the diver caused appreciable bias with the transect method but not with the point count method, because of underlying assumptions about how the two methods were implemented. Performance of methods was quantified with the root mean squared error RMSE (combined measure of bias and variability) and was dependent on the ratio of sampling times per dive for each method. From assumed sampling times for the point count and transect methods, the point count method performed better than the transect method, but different results could be obtained under different sampling protocols. Nevertheless, the simulation approach offers an efficient means to evaluate sampling methods in conjunction with actual field experiments. (C) 1997 Elsevier Science B.V.
\end{abstract}

Keywords: Visual census; Transect; Point count; Performance; Bias; Reef; Diver survey

\section{Introduction}

Underwater visual census (UVC) methods have

\footnotetext{
* Corresponding author. Fax: + 618 94473062; e-mail: rwatson@omen.com.au
}

been used to estimate the densities of reef fish since the 1950s (Brock, 1954; Harmelin-Vivien et al., 1985). These methods are usually quick and inexpensive to employ, and non-destructive in nature. These advantages have led to their adoption in many coastal resource studies (Russ, 1985; Samoilys, 1988; Samoilys and Carlos, 1991). 
There is continuing concern over the accuracy of UVC density estimates (Brock, 1982; Sale and Sharp, 1983; Andrew and Mapstone, 1987), but in general these have been difficult to test. Several sources of bias have been recognised including: observer error, observer presence and speed, and fish detectability (Thresher and Gunn, 1986; Lincoln Smith, 1988), but in practice these are rarely measurable.

Field conditions where UVC methods are employed vary considerably and practitioners have long argued over the relative advantages of the various methods. The transect and point count methods can both produce unbiased and precise estimates of fish abundance under the right conditions - but at what cost? It is very difficult to measure the bias and precision of these methods in the field, and to observe how these are improved with additional samples or resources. This information would be useful in the design of studies using UVC, and would allow the more efficient use of sampling resources such as diver time. Controls on daily dive time and the number of diver ascents recently introduced on scientific diving in Australia and elsewhere in response to health concerns, mean that these resources are more precious than ever.

There are two general methods for testing UVC bias, the first is through field experiment (McCormick and Choat, 1987; Lincoln Smith, 1989), and the second is through computer simulation (Turnock and Quinn II, 1991; Watson et al., 1995). The simulation study of diver transects by Watson et al. (1995) demonstrated and quantified bias associated with density estimates introduced by the non-random movement of fish.

Our purpose in this study is to use the simulation approach to make a comparative study of the performance (as it relates to the reduction of bias and variability with diver time) of the transect and point count UVC methods under a range of conditions which might be encountered in field studies of reef fish.

\section{Methods and Materials}

\subsection{Model description}

The simulation program Reefex (Watson et al., 1995) was used to model the transect and point count UVC processes in three experimental situations described below. The Reefex simulation model provides a two-dimensional, animated simulation of the visual census process, depicting both the movement of fish and of the diver.

Within the Reefex program, groups of fish can be defined, which represent different species or different size groups within a species. For each group the user can control: fish density, the maximum distance from the diver that they can be seen (visibility), and the minimum distance that they will allow the diver to approach them or vice versa (approach distance). The user describes behavioural states which control individual fish movement and other responses for each fish group. For each behavioural state the user specifies: the probability of entering the state, an associated swimming speed of the fish, and the probability of moving in each of four directions in a horizontal plane $\left(0,90,180\right.$ and $\left.270^{\circ}\right)$. As an example, for a fish group we might define three possible behavioural states: 'stationary', 'random-movement', and 'cruising'. We might specify that any individual fish is in the 'stationary' state for $50 \%$ of the time, in the 'random-movement' state for $30 \%$ of the time, and the balance in the 'cruising' state. For each of these three states we could then describe the direction and speed of movement. We would not move fish while they are in the 'stationary' state, however, while a fish is in the 'random-movement' state we might allow movement with equal probability in each of the four directions at $2 \mathrm{~m} \mathrm{~min}^{-1}$, and for the 'cruising' state we could allow a greater probability of movement at $90^{\circ}$ than in the other directions and at a faster speed, i.e. $4 \mathrm{~m} \mathrm{~min}^{-1}$.

The length of the time steps used in the simulation can be specified. At each time step the behavioural state and the subsequent movement of each individual fish in each group is determined randomly by user-defined probability distributions. At each time step the new positions of the diver and the fish can be shown. 
The total area of the reef represented in the simulation can be defined, as can the dimensions of the strip transect. All or only a portion of this total reef can be shown depending on the display scale chosen. For reference, grid lines can be displayed in any scale. If fish move out of the defined reef area, they are removed from the simulation, but are replaced on the opposite boundary so that the specified densities within the simulated reef are maintained.

The model allows for a count-saturation level to be defined for each fish group, that is the maximum number of fish in that group that a diver can count at any one time step. It is possible to create conditions where there is an established counting hierarchy within the fish groups, that is, the diver will attempt to count all of the highest priority species, and if time permits, proceed to the next group and so on. It is also possible to introduce error in the counting process either through allowing some fish to be missed or wrongly identified.

Ranges of parameter values were chosen to describe conditions described by Samoilys and Carlos (1992) in surveys of larger reef fish species such as Plectropomus leopardus (coral trout). The simulated UVC counts take place within a modelled universe of size $250 \times 100 \mathrm{~m}$ using $10 \mathrm{~s}$ time steps. No limits were placed on a diver's ability to recognise or record fish sightings. Fish were stationary (with no net movement with respect to the diver) in all but experiment 3. Fish speeds refer to the net fish speed of all individuals combined. The assumed fish density was $70 \mathrm{ha}^{-1}$ (except experiment 1 where a range was used). Fish distribution was random with respect to the sampling area and the diver.

The transect method was modelled as shown in Fig. 1a. The simulated diver moved along the transect (dimensions were $5 \times 50 \mathrm{~m}$ ) in direction $D$ at speed $n\left(7 \mathrm{~m} \mathrm{~s}^{-1}\right)$. Counts were made of fish within the transect's width when the simulated diver encountered them. Fish seen entering the transect within the diver's view (a circle with radius of visibility $V$ of $10 \mathrm{~m}$ ) were not counted. Fish movement simulated in experiment 3 was in a direction approaching the diver head-on (in the opposite direction of vector $D$, Fig. 1a). In other experiments the simulated fish were stationary. Only fish seen within the confines of the transect when the diver approached were counted. The shaded area of Fig. 1a represents the area where fish are counted during each time step. The standard number of dives $d$ per survey was 15 (except experiment 2), and the assumed combined set-up and swim time was 17 min (Samoilys and Carlos, 1992). This latter assumption was investigated in greater detail below.

The point count method was modelled as shown in Fig. 1b. The simulated diver approaches from the surface and immediately counts all mobile species within a path transcribing a circular area of the bottom with a $10 \mathrm{~m}$ radius (shaded area of Fig. 1b). The diver continues to descend and spends the balance of the dive time searching the circular area of the bottom for more sedentary and cryptic species (all fish modelled were visible within $10 \mathrm{~m}$ ). It is assumed that all fish present within the circle are seen and counted, and that no fish which enter the circle after the count starts are counted. The standard number of dives per survey was 15 (except experiment 2) and the assumed combined set-up and swim time was 12 min (Samoilys and Carlos, 1992).

For each of the experiments described below, 500 replicates were performed, and for each, a new population of fish was generated.

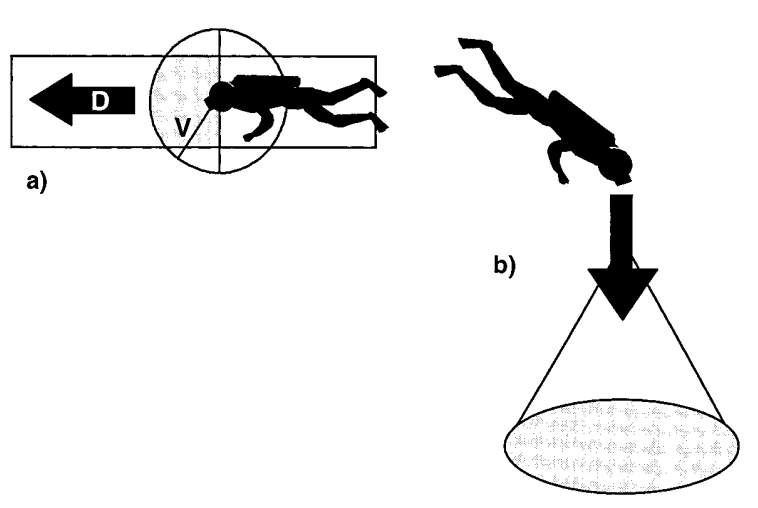

Fig. 1. Illustration for the transect (a) and point count (b) methods. Diver movement is indicated by arrow $(D)$. Radius of visibility $(V)$ indicated as circle for transect method. Fish are counted within the shaded areas. 


\subsection{Experiment 1 (fish density)}

As referred to in the model description except that fish density was varied from 10 to $150 \mathrm{ha}^{-1}$ by steps of 10 . The standard fish speed $\left(0 \mathrm{~m} \mathrm{~s}^{-1}\right)$ and numbers of dives per sample (15) were used in each replicate.

\subsection{Experiment 2 (sample size)}

As referred to in the model description except the number of dives were varied from 5 to 25 per sample by steps of 5 for each replicate. The standard fish speed $\left(0 \mathrm{~m} \mathrm{~s}^{-1}\right)$ and density (70 $\mathrm{ha}^{-1}$ ) was assumed.

\subsection{Experiment 3 (fish speed)}

As referred to in the model description except that fish speed was not assumed to be stationary but instead fish approached the diver head-on at speeds ranging from 0 to $20 \mathrm{~m} \mathrm{~s}^{-1}$ by steps of 2 . The standard fish density $\left(70 \mathrm{ha}^{-1}\right)$ and number of dives per sample (15) were used in each replicate.

\subsection{Performance calculations}

The subscripts $T$ and $P$ are used for transect and point count, respectively. Each replicate consisted of $d$ dives, with a sampled area of $a_{T}=5 \times$ $50=250 \mathrm{~m}^{2}$ for transects and $a_{P}=\pi \times 10^{2}=314$ $\mathrm{m}^{2}$ for point counts. If $n_{i}$ is the number of fish seen over the $d$ dives, then the standard estimator of abundance for both methods is

$\hat{D}_{i}=n_{i} /\left(d a_{i}\right), \quad i=T, P$

This estimator can also be expressed in terms of minutes of dive time. If $c_{i}$ is the number of minutes per dive $\left(M_{T}=17, m_{P}=12\right)$, then the total number of minutes per replication is $m_{i}=$ $d c_{i}$. The estimator (1) can then be written

$\hat{D}_{i}=c_{i} n_{i} /\left(m_{i} a_{i}\right), \quad i=T, P$

If animals are distributed randomly, then $n$ has a Poisson distribution with variance $\operatorname{var}(n)$ equal to its expected value $E(n)$. If animals do not move, then both transect and point counts are unbiased (see results below), so that the variance is equal to the mean squared error, a combined measure of bias and variability defined below. From Eq. (1),

$\operatorname{var}(\hat{D})=\operatorname{MSE}(\hat{D})=E\left(n_{i}\right) /\left(d a_{i}\right)^{2}=D /\left(d a_{i}\right)$,

since $E(n)=d a_{i} D$

In terms of minutes of dive time as in Eq. (2), Eq. (3) can be written as:

$\operatorname{var}(\hat{D})=\operatorname{MSE}(\hat{D})=c_{i} D /\left(a_{i} m_{i}\right)$

Hence the ratio of MSEs for transect and point counts when unbiased estimators are used is

$\frac{\operatorname{MSE}\left(\hat{D}_{T}\right)}{\operatorname{MSE}\left(\hat{D}_{p}\right)}=\sqrt{\frac{c_{T} m_{p} a_{p}}{c_{p} m_{T} a_{T} v}}$

From each set of 500 replications, a number of summary statistics was calculated, including average density, standard error (S.E.) (calculated as the empirical S.D. across replications), skewness, and kurtosis. Skewness and kurtosis were calculated to assess whether the distribution of the estimator of density was approximately normally distributed, an important consideration in calculating confidence intervals and conducting statistical tests. (Critical values were calculated by generating 3000 replications of 500 normal random numbers and finding the 2.5 and $97.5 \%$ points, resulting in a range of non-significance of -0.35 to 0.34 for skewness and -0.76 to 0.93 for kurtosis.) Estimator bias $(B)$ was estimated as average density minus true density, and judged unimportant if it was less than 0.25 times the standard error, in accordance with other studies (e.g. Buckland et al. 1993). As a combined measure of bias and variability, the empirical root mean squared error (RMSE) was calculated as

$\mathrm{RMSE}=\sqrt{\text { S.E. }^{2}+B^{2}}$

For each experiment, summary statistics were compared between transect and point counts. Because the unit sample area for point counts was higher than for transects, the same number of dives provided less sampling for transects. We set the simulation up this way, because the unit sampling areas are those commonly employed in applications. Nevertheless, for a more valid 
Table 1

Summary statistics as a function of true density $D$ for experiment 1 from 500 replications for the transect method $(T)$ and the point count method $(P)$

\begin{tabular}{|c|c|c|c|c|c|c|c|c|c|}
\hline$D$ & $\begin{array}{l}B \\
T\end{array}$ & $\begin{array}{l}B \\
P\end{array}$ & $\begin{array}{l}\text { RMSE } \\
T\end{array}$ & $\begin{array}{l}\text { RMSE* } \\
T\end{array}$ & $\begin{array}{l}\text { RMSE } \\
P\end{array}$ & $\begin{array}{l}\gamma_{1} \\
T\end{array}$ & $\begin{array}{l}\gamma_{1} \\
P\end{array}$ & $\begin{array}{l}\gamma_{2} \\
T\end{array}$ & $\begin{array}{l}\gamma_{2} \\
P\end{array}$ \\
\hline 10 & 0.0 & -0.3 & 5.4 & 4.8 & 4.6 & 0.70 & 0.48 & 0.75 & 0.16 \\
\hline 20 & 0.4 & 0.2 & 7.2 & 6.4 & 6.5 & 0.30 & 0.33 & -0.21 & 0.42 \\
\hline 30 & 0.0 & -0.1 & 9.1 & 8.1 & 8.1 & 0.52 & 0.42 & 0.99 & 0.49 \\
\hline 40 & 0.1 & -0.6 & 10.6 & 9.4 & 8.8 & 0.31 & 0.08 & -0.12 & -0.03 \\
\hline 50 & 1.1 & 0.0 & 11.8 & 10.6 & 10.0 & 0.29 & 0.15 & 0.32 & -0.20 \\
\hline 60 & 0.3 & -0.2 & 12.9 & 11.4 & 11.4 & 0.24 & 0.36 & 0.11 & 0.19 \\
\hline 70 & 2.1 & -0.8 & 13.2 & 11.8 & 12.1 & 0.21 & 0.37 & 0.21 & -0.17 \\
\hline 80 & 0.3 & -1.0 & 15.0 & 13.3 & 12.7 & 0.05 & 0.12 & -0.21 & 0.11 \\
\hline 90 & -0.1 & 1.4 & 15.1 & 13.5 & 14.2 & 0.36 & -0.01 & 0.46 & 0.17 \\
\hline 100 & -0.8 & 0.3 & 15.2 & 13.6 & 14.9 & 0.14 & 0.15 & 0.03 & -0.26 \\
\hline 110 & 0.7 & -1.2 & 16.3 & 14.6 & 15.5 & 0.06 & 0.15 & -0.21 & -0.31 \\
\hline 120 & 0.3 & -0.5 & 17.7 & 15.8 & 16.6 & 0.25 & 0.03 & -0.19 & -0.30 \\
\hline 130 & -0.1 & 0.9 & 17.7 & 15.8 & 16.2 & 0.28 & 0.03 & -0.03 & 0.19 \\
\hline 140 & 2.3 & -0.5 & 20.1 & 17.9 & 17.4 & 0.17 & 0.16 & 0.17 & -0.05 \\
\hline 150 & 0.7 & -0.4 & 19.8 & 17.7 & 16.2 & 0.18 & 0.01 & -0.26 & 0.30 \\
\hline
\end{tabular}

$B$, estimated bias; RMSE, root mean squared error (see text for definition); $\gamma_{1}$, normalised skewness; $\gamma_{2}$, normalised kurtosis. For a normal distribution, $\gamma_{1}$ and $\gamma_{2}$ should not be statistically different from 0 . In this experiment, fish do not move and the number of dives $d$ is 15 .

Bold values for bias, skewness or kurtosis are significantly different from 0 (in the case of bias, $<0.5$ the RMSE).

comparison between methods, we also determined an adjusted variance $\mathrm{SE}^{* 2}$ (and consequently RMSE) for the transects, had the same amount of area been sampled (essentially 12.5\% more area than originally done) as with the point count method. In Eq. (2), the variance is inversely proportional to sample area, so the variance was adjusted by the ratio of sampled areas. The corresponding RMSE from Eq. (6) is denoted RMSE*.

\section{Results}

\subsection{Experiment 1 (fish density)}

Table 1 gives summary statistics for bias, RMSE, skewness, and kurtosis. Over the range of fish density from 10 to $150 \mathrm{ha}^{-1}$, neither method had appreciable bias in the estimator of density. The empirical RMSE (and also variance, not shown) increased linearly with the square root of density, as expected from Eqs. (2) or (3), and the empirical RMSE was close to the theoretical, as illustrated in Fig. 2. The adjusted RMSE* from transects and the RMSE from point counts were nearly identical (Fig. 3). Significant skewness in the distribution of estimated density was occasionally present, usually at lower densities. There was significant kurtosis only for one density in the point count method (probably due to chance).

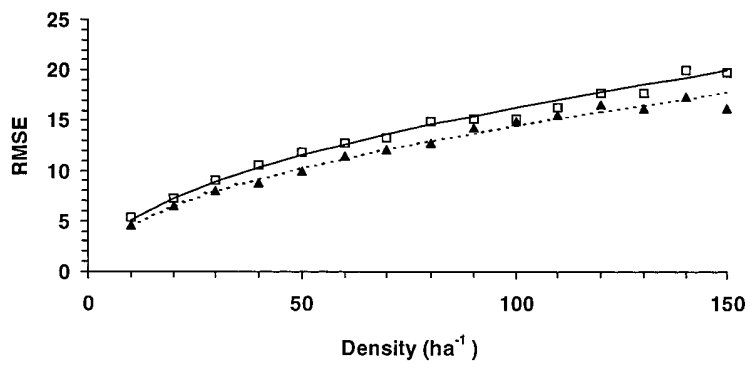

Fig. 2. Root mean squared error (RMSE) associated with fish density estimates using the transect (theoretical, solid line; empirical, open squares) and point count methods (theoretical, dashed line; empirical, closed triangles) for a range of simulated fish densities (experiment 1). 


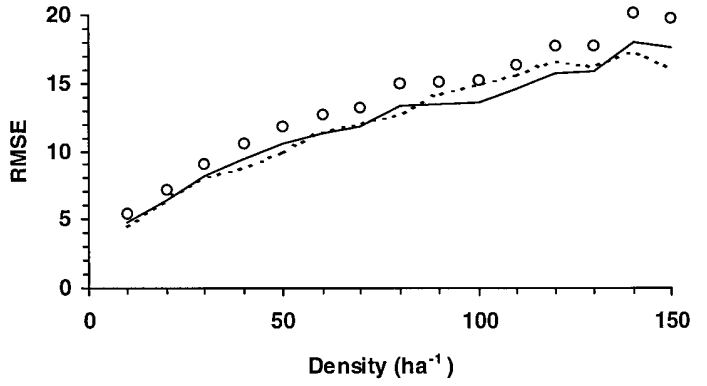

Fig. 3. Root mean squared error (RMSE) associated with fish density estimates using the transect (unadjusted, open circles; adjusted, solid line) and point count methods (dashed line) for a range of simulated fish densities (experiment 1)

\subsection{Experiment 2 (sample size)}

Over the range of dive number $d$ from 5 to 25 , neither method had appreciable bias in the estimator of density (Table 2). The empirical RMSE was inversely related to square root of dive number, as expected from Eqs. (2) or (3). The adjusted RMSE* from transects and the RMSE from point counts were nearly identical (Fig. 4). There is also an inverse relationship with square root of minutes of dive time, which is proportional to dive number. There was no consistently significant skewness in the distribution of density and no significant kurtosis.

\subsection{Experiment 3 (fish speed)}

Over the range of fish speed head-on to the diver at speeds ranging from 0 to $20 \mathrm{~m} \mathrm{~s}^{-1}$, the point count method had no appreciable bias in the estimator of density, but the transect method had a bias that increased nearly linearly with speed (Table 3). The empirical variance appeared constant as a function of speed for the point count method, and hence its empirical RMSE was also constant. The RMSE for transects was a linear function of speed and almost solely due to bias beyond a fish speed of $2 \mathrm{~m} \mathrm{~s}^{-1}$ (Fig. 5). There was no significant skewness or kurtosis.

\subsection{Performance as a function of unit time per dive}

From experiment 2, the RMSE is plotted against sampling time for each method (Fig. 6). For the given parameters above for sample area and sampling time per dive, the point count method achieves lower RMSE with a lower amount of total sampling time. It is possible to explore the sensitivity of this result to these parameters. Supposing that the same amount of total time would be available for conducting each of the methods $\left(m_{T}=m_{P}\right)$ and that the standard sampling areas above would be used, then one can determine the ratio of RMSEs as a function of the ratio of sampling times per dive with Eq. (5). As shown in Fig. 7, as the ratio of transect time to point count time per dive increases, the ratio of the RMSEs increases from below 1 to above 1 . The current dive time ratio is $17 / 12=1.42$ and yields an RMSE ratio of 1.34 . If the dive time ratio were 0.8 , then the RMSEs would be equal.

\section{Discussion}

The results show that the transect and point count methods perform equivalently in terms of bias and variability when fish do not move, the amount of sampled area is the same, and all fish within the sampled area are observed. Furthermore, it does not matter what size the individual sample areas are nor what the number of dives is, because estimator variability is a function only of the total sampled area. However, if fish have a clustered distribution, then this issue of sample design could be important, although this was not investigated in this study.

When fish move, the nature of the movement is critical in determining the amount of bias in an estimator. In the transect method, we studied the worst case scenario, where there is directional movement toward the observer, and reconfirmed the results of Watson et al. (1995) that a positive bias is induced in the density estimate. If fish move perpendicular to the observer, then little bias would be expected, and if fish move away from the observer, a negative bias would be ex- 
Table 2

Summary statistics as a function of the number of dives $d$ for experiment 2 from 500 replications for the transect method (T) and the point count method $(P)$ with notation as in Table 1

\begin{tabular}{|c|c|c|c|c|c|c|c|c|c|}
\hline$d$ & $\begin{array}{l}B \\
T\end{array}$ & $\begin{array}{l}B \\
P\end{array}$ & $\begin{array}{l}\text { RMSE } \\
T\end{array}$ & $\begin{array}{l}\text { RMSE* } \\
T\end{array}$ & $\begin{array}{l}\text { RMSE } \\
P\end{array}$ & $\begin{array}{l}\gamma_{1} \\
T\end{array}$ & $\begin{array}{l}\gamma_{1} \\
P\end{array}$ & $\begin{array}{l}\gamma_{2} \\
T\end{array}$ & $\begin{array}{l}\gamma_{2} \\
P\end{array}$ \\
\hline 5 & -0.2 & -0.5 & 24.8 & 22.1 & 21.6 & 0.48 & 0.18 & 0.09 & -0.15 \\
\hline 6 & -0.9 & 0.1 & 21.2 & 18.9 & 18.8 & 0.32 & 0.24 & 0.33 & 0.28 \\
\hline 7 & 0.7 & 0.2 & 19.9 & 17.7 & 17.2 & 0.24 & 0.29 & 0.02 & 0.25 \\
\hline 8 & -0.3 & 1.9 & 19.1 & 17.0 & 16.2 & 0.24 & 0.23 & -0.05 & 0.58 \\
\hline 9 & 0.4 & -0.6 & 17.3 & 15.4 & 15.9 & 0.27 & 0.31 & -0.13 & 0.01 \\
\hline 10 & 0.9 & 1.1 & 16.7 & 14.9 & 14.5 & 0.21 & 0.26 & 0.07 & 0.26 \\
\hline 11 & 1.0 & 0.6 & 15.9 & 14.2 & 14.2 & 0.10 & 0.15 & -0.27 & -0.23 \\
\hline 12 & -0.8 & -0.7 & 15.1 & 13.4 & 12.8 & 0.21 & 0.19 & 0.00 & -0.10 \\
\hline 13 & 0.6 & -0.3 & 15.7 & 14.0 & 13.3 & 0.18 & 0.18 & -0.17 & -0.03 \\
\hline 14 & 0.8 & 0.2 & 13.9 & 12.4 & 12.4 & 0.02 & 0.14 & 0.24 & -0.35 \\
\hline 15 & -0.3 & 0.3 & 13.2 & 11.7 & 11.8 & 0.15 & 0.45 & 0.07 & 0.40 \\
\hline 16 & 0.3 & 0.0 & 12.9 & 11.5 & 10.8 & 0.20 & 0.10 & -0.12 & 0.22 \\
\hline 17 & -0.2 & 0.0 & 12.5 & 11.2 & 12.0 & 0.05 & 0.09 & -0.14 & -0.10 \\
\hline 18 & -0.6 & -0.2 & 12.6 & 11.2 & 11.3 & 0.24 & 0.11 & 0.16 & -0.14 \\
\hline 19 & -0.2 & -0.5 & 12.1 & 10.8 & 10.8 & 0.09 & 0.28 & -0.10 & 0.11 \\
\hline 20 & -0.4 & 0.3 & 11.6 & 10.4 & 10.6 & 0.12 & 0.21 & -0.38 & -0.09 \\
\hline 21 & -0.3 & 0.0 & 11.5 & 10.3 & 10.6 & 0.26 & 0.32 & 0.15 & 0.25 \\
\hline 22 & -0.6 & 0.6 & 11.1 & 9.9 & 10.0 & 0.24 & 0.18 & 0.20 & 0.16 \\
\hline 23 & 0.0 & -0.1 & 11.1 & 9.9 & 9.7 & 0.16 & 0.12 & 0.07 & -0.06 \\
\hline 24 & -0.3 & 0.4 & 10.3 & 9.1 & 9.5 & 0.22 & 0.30 & -0.05 & 0.12 \\
\hline 25 & 0.3 & -1.1 & 10.0 & 8.9 & 9.7 & -0.06 & 0.13 & -0.01 & -0.07 \\
\hline
\end{tabular}

In this experiment, fish do not move, and the true density $D$ is $70 \mathrm{ha}^{-1}$.

Bold values for bias, skewness or kurtosis are significantly different from 0 .

pected. In the absence of movement information, transect direction should be randomly chosen to reduce bias, however, when movement is known to exist transects should be orientated perpendicular to the direction of moment. We modelled the point count method as being able to detect and count all motile fish within a $10 \mathrm{~m}$ radius instan-

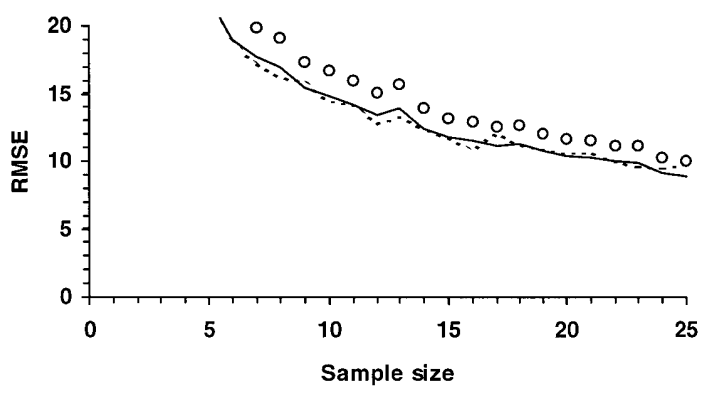

Fig. 4. Root mean squared error (RMSE) associated with fish density estimates using the transect (circle, unadjusted for sample area; solid line, adjusted) and point count methods (dashed line) using a range of sample sizes (experiment 2). taneously, and to spread remaining time in searching for cryptic and hidden fish. As a result, the estimate of density was unbiased even when fish moved. However, if fish are attracted into or even randomly move into the sample circle and are counted, then a similar bias as found in the transect method would be expected.

We did not study the effects of incomplete detection, but it is well known that density is underestimated if some fish are not seen (Seber, 1982; Thompson, 1992). The size of the sample area must be small enough to avoid incomplete detection. Otherwise, distances should be measured to the fish and used in conjunction with the line transect method (Buckland et al., 1993).

The skewness and kurtosis statistics show that for the most part, the distribution of estimated density is close to a normal distribution. At any rate, the use of standard confidence interval methods appears appropriate, except for small sample sizes or densities. 
Table 3

Summary statistics as a function of fish speed $v$ for experiment 2 from 500 replications for the transect method $(T)$ and the point count method $(P)$, with notation as in Table 1

\begin{tabular}{|c|c|c|c|c|c|c|c|c|c|}
\hline \multirow[t]{2}{*}{$v$} & $B$ & $B$ & RMSE & RMSE* & RMSE & $\gamma_{1}$ & $\gamma_{1}$ & $\gamma_{2}$ & $\gamma_{2}$ \\
\hline & $T$ & $P$ & $T$ & $T$ & $P$ & $T$ & $P$ & $T$ & $P$ \\
\hline 0 & 1.1 & -0.5 & 14.1 & 12.6 & 12.0 & 0.25 & 0.16 & 0.34 & -0.02 \\
\hline 2 & 15.8 & -0.9 & 21.0 & 20.1 & 12.6 & -0.18 & 0.23 & -0.27 & 0.43 \\
\hline 4 & 32.4 & -0.5 & 36.3 & 35.5 & 11.9 & 0.23 & -0.03 & -0.07 & -0.06 \\
\hline 6 & 47.4 & -0.3 & 50.4 & 49.8 & 11.6 & -0.01 & 0.08 & -0.25 & -0.20 \\
\hline 8 & 64.1 & 0.5 & 66.9 & 66.3 & 12.6 & 0.05 & 0.19 & 0.16 & -0.10 \\
\hline 10 & 80.6 & -0.8 & 82.9 & 82.4 & 12.4 & 0.12 & 0.07 & -0.13 & 0.16 \\
\hline 12 & 95.0 & 0.1 & 97.1 & 96.6 & 11.6 & 0.02 & 0.18 & -0.13 & -0.18 \\
\hline 14 & 112.4 & -0.3 & 114.3 & 113.9 & 12.5 & 0.12 & 0.31 & -0.27 & 0.16 \\
\hline 16 & 129.0 & -0.6 & 131.1 & 130.7 & 12.2 & 0.10 & 0.20 & -0.12 & -0.08 \\
\hline 18 & 142.8 & 0.2 & 144.8 & 144.4 & 11.9 & 0.18 & 0.11 & -0.05 & 0.02 \\
\hline 20 & 159.0 & 0.1 & 160.9 & 160.5 & 11.9 & 0.13 & 0.20 & 0.16 & 0.26 \\
\hline
\end{tabular}

In this experiment, the number of dives $d$ is 15 , and the true density $D$ is 70 ha $^{-1}$. Bold values for bias, skewness or kurtosis are significantly different from 0 .

We evaluated the performance of the two methods with respect to logistical efficiency by using literature values for the amount of sampling time per dive (12 versus 17 min for point counts versus transects). Because the point count sample area per dive is larger than that for transects, and the amount of time is shorter as well, it comes as no surprise that the point count method was judged superior in this situation. The use of a combination of point count and transect methods offers some advantages when conditions, fish movement, and visibility vary.

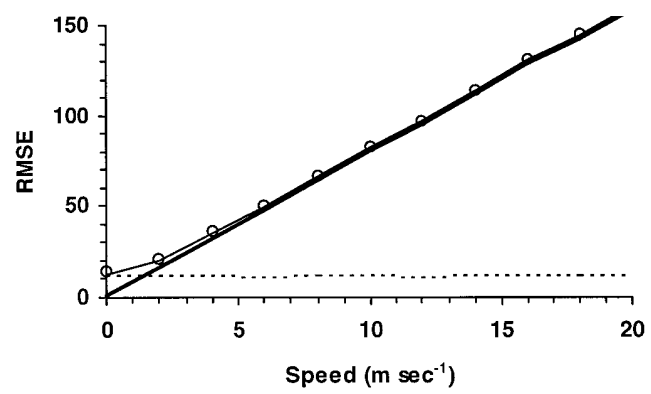

Fig. 5. Root mean squared error (RMSE) associated with fish density estimates using the transect (circle, unadjusted for sample area, solid line, adjusted) and point count methods (dashed line) for a range of net fish speeds (experiment 3). Bias associated with the transect method is shown as a thick solid line.
Performing a series of transects at different speeds may offer some advantages when several species which differ in their speed and visibility must be surveyed together (Lincoln Smith, 1988; 1989). In general, surveys which cover larger areas would be more efficient because they reduce set-up time as a proportion of sampling time. Limited visibility may in practice, however, constrain this approach to the transect method.

Practical considerations which our study raises with regard to survey design and choice of methodology relate mostly to the relative performance of the two methods under field conditions, and the risk of biased estimates caused primarily through fish movement. Initial field observations, or even literature searches on the species of interest, can help establish the risk of bias. If this risk is deemed significant, then the survey design could include transects orientated to reduce this effect, or the use of point counts which by their nature are not affected. The best mixture of methodology will depend on the relative cost (for example diver time) per area surveyed and other logistical considerations. The ultimate judgment, however, can only be made after field experimentation is done under a variety of different conditions, and we encourage further work in this area. 


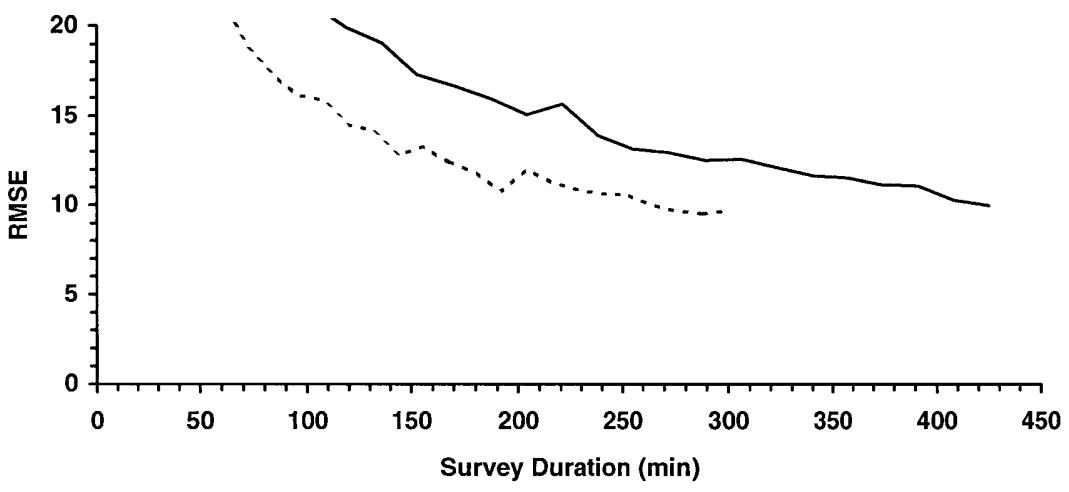

Fig. 6. Root mean squared error (RMSE) associated with fish density estimates using the transect (solid line) and point count methods (dashed line) as a function of survey duration.

\section{Acknowledgements}

The authors thank Norm Hall for his suggestions, and gratefully acknowledge M. Samoilys and M. Lincoln Smith for providing valuable practical insights into the application of transect and point count methods. The contributions of two anonymous referees is also acknowledged. This work was in part supported by the Fisheries Department of Western Australia and the School of Fisheries and Ocean Sciences, University of Alaska, Fairbanks, and is an extension to work initiated under an Australian Centre for International Agricultural Research project.

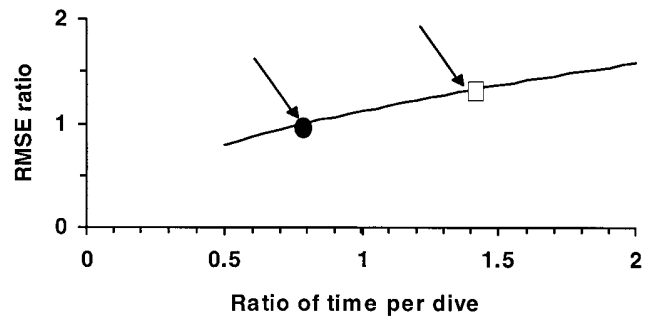

Fig. 7. Ratio of root mean squared error (RMSE) associated with fish density estimates as a function of the ratio of sampling time per dive (in both cases the ratio is transect to point count). Ratio of sampling time assumed is marked by an open rectangle. The filled circle marks a point where the MSE ratio is 1 .

\section{References}

Andrew, N.L., Mapstone, B.D., 1987. Sampling and the description of spatial pattern in marine ecology. Oceanogr. Mar. Biol. Annu. Rev. 25, 39-90.

Brock, V.E., 1954. A preliminary report on a method of estimating reef fish populations. J. Wildl. Manage. 18, 297-308.

Brock, R.E., 1982. A critique of the visual census method for assessing coral reef fish populations. Bull. Mar. Sci. 32, 269-276.

Buckland, S.T., Anderson, D.R., Burnham, K.P., Laake, J.L., 1993. Distance Sampling, Chapman and Hall, London. 446 pp.

Harmelin-Vivien, M.L., Harmelin, J.G., Chauvet, C., Duval, C., Galzin, R., Lejeune, P., Barnabé, G., Blanc, F., Ghevalier, R., Duclerc, J., Lasserre, G., 1985. Evaluation visuelle des peuplements et populations de poissons: methodes et problemes. Rev. Ecol. (Terre Vie) 40, 468-539.

Lincoln Smith, M.P., 1988. Effects of observer swimming speed on sample counts of temperate rocky reef fish assemblages. Mar. Ecol. Prog. Ser. 43, 223-231.

Lincoln Smith, M.P., 1989. Improving multispecies rocky reef fish census by counting different groups of species using different procedures. Environ. Biol. Fish. 26, 29-37.

McCormick, M.I., Choat, J.H., 1987. Estimating total abundance of a large temperate-reef fish using visual trip-transects. Mar. Biol. 96, 469-478.

Russ, G., 1985. Effects of protective management on coral reef fish in the Central Philippines. In: 5th International Coral Reef Congress, Tahiti, vol. 4 Museum-EPHE, Tahiti, pp. 219-224.

Sale, P.F., Sharp, B.J., 1983. Correction for bias in visual transect censuses of coral reef fish. Coral Reefs 2, 37-42.

Samoilys, M.A., 1988. Abundance and species richness of coral reef fish on the Kenya coast: the effects of protective management and fishing. In: Proceedings of the 6th Coral Reef Symposium, Townsville, Australia, vol. 2, pp. 261266. 
Samoilys, M., Carlos, G., 1991. A survey of reef fish stocks in Western Samoa: application of underwater visual census methods for fisheries personnel. A report prepared for the Forum Fisheries Agency, Honiara, Solomon Islands, and the Fisheries Division, Western Samoa, 26 pp.

Samoilys, M., Carlos, G., 1992. Development of an underwater visual census method for assessing shallow water reef fish stocks in the south west pacific. mimeo. Final report project PN 8545. Australian Centre for International Agricultural Research, 100 pp.

Seber, G.A.F., 1982. The Estimation of Animal Abundance, Griffin, London.
Thompson, S.K., 1992. Sampling. Wiley, New York, 343 pp.

Thresher, R.E., Gunn, J.S., 1986. Comparative analysis of visual census techniques for highly mobile, reef-associated piscivores (Carangidae). Environ. Biol. Fish. 17, $93-116$.

Turnock, B.J., Quinn II, T.J., 1991. The effect of responsive movement on abundance estimation using line transect sampling. Biometrics 47, 701-715.

Watson, R.A., Carlos, G.M., Samoilys, M.A., 1995. Bias introduced by the non-random movement of fish in visual transect surveys. Ecol. Model. 77, 205-214. 